\title{
EULAR recommendations for the management of knee osteoarthritis: report of a task force of the Standing Committee for International Clinical Studies Including Therapeutic Trials (ESCISIT)
}

\author{
A Pendleton, N Arden, M Dougados, M Doherty, B Bannwarth, J W J Bijlsma, F Cluzeau, C Cooper, P A Dieppe, \\ K-P Günther, H J Hauselmann, G Herrero-Beaumont, P M Kaklamanis, B Leeb, M Lequesne, S Lohmander, \\ B Mazieres, E-M Mola, K Pavelka, U Serni, B Swoboda, A A Verbruggen, G Weseloh, I Zimmermann-Gorska
}

\begin{abstract}
Background-Osteoarthritis (OA) is the most common joint disease encountered throughout Europe. A task force for the EULAR Standing Committee for Clinical Trials met in 1998 to determine the methodological and logistical approach required for the development of evidence based guidelines for treatment of knee OA. The guidelines were restricted to cover all currently available treatments for knee OA diagnosed either clinically and/or radiographically affecting any compartment of the knee.

Methods-The first stage was the selection of treatment modalities to be considered. The second stage comprised a search of the electronic databases Medline and Embase using a combination of subject headings and keywords. All European language publications in the form of systematic reviews, metaanalyses, randomised controlled trials, controlled trials, and observational studies were included. During stage three all the relevant studies were quality scored. The summary statistics for validated outcome measures, when available, were recorded and, where practical, the numbers needed to treat and the effect size for each treatment were calculated. In the fourth stage key clinical propositions were determined by expert consensus employing a Delphi approach. The final stage ranked these propositions according to the available evidence. A second set of propositions relating to a future research agenda was determined by expert consensus using a Delphi approach.

Results-Over 2400 English language publications and 400 non-English language publications were identified. Seven hundred and forty four studies presented outcome data of the effects of specific treatments on knee OA. Quantitative analysis of treatment effect was possible in only 61 studies. Recommendations for the management of knee OA based on currently available data and expert opinion are presented. Proposals for a future research agenda are highlighted.

Conclusions-These are the first clinical guidelines on knee OA to combine an evidence based approach and a consensus approach across a wide range of treatment modalities. It is apparent that certain clinical propositions are supported by substantial research based evidence, while others are not. There is thus an urgent need for future well designed trials to consider key clinical questions.

(Ann Rheum Dis 2000;59:936-944)
\end{abstract}

Osteoarthritis (OA) is by far the most common disease to affect synovial joints. It is a major cause of musculoskeletal pain, the single most important cause of disability and handicap from arthritis, and an important community healthcare burden. OA is strongly associated with aging and, with the increasing proportion of elderly in Western populations, large joint OA, particularly of the knee, will become an even more important healthcare challenge.

Multiple genetic, constitutional, and environmental factors may contribute to the development and variable outcome of OA. OA of the knees is particularly common, with radiographic $\mathrm{OA}$ of the tibiofemoral compartment occurring in $5-15 \%$ of people aged $35-74$ years. The condition is often associated with pain and disability and symptomatic subjects often seek medical advice. Treatment aims at $(a)$ educating the patient about OA, $(b)$ alleviating pain, $(c)$ optimising and maintaining function, and (d) preventing or retarding progression of adverse structural change affecting the joint tissues (cartilage, bone, ligament, muscle). Current treatments include a wide range of non-pharmacological, pharmacological, and surgical modalities. Evidence to support the effectiveness of individual treatments, however, is variable.

Clinical guidelines were defined in 1995 by the Institute of Medicine in Washington as "Systematically developed statements to assist practitioner and patient decisions about appropriate health care for specific clinical conditions." Using these principles, guidelines on the management of knee OA have been published by the American College for Rheumatology ${ }^{1}$ and the Royal College of Physicians. ${ }^{2}$ However, such guidelines primarily represent consensus statements from expert panels. The type and strength of evidence to support such guidelines remain unclear. In 1998 EULAR commissioned a steering committee to review the available research evidence for treatments of knee $\mathrm{OA}$, to develop guidelines relating to clinical issues in OA management, and to indicate clearly the level of evidence to support individual statements. It was felt that the development of such evidence based guidelines for the management of knee OA might lead to improvements in patient care and to more uniform treatment approaches throughout Europe.

\section{Methods}

MEMBERSHIP OF GUIDELINES STEERING COMMITTEE

The initiative endorsed by ESCISIT involved a committee of 21 experts in the field of osteoarthritis (18 rheumatologists, three orthopaedic surgeons) representing 12 different 
Table 1 Interventions selected for assessment in the treatment of knee osteoarthritis

\begin{tabular}{|c|c|c|}
\hline Non-pharmacological & Pharmacological & Surgical \\
\hline Patient education & NSAIDs ${ }^{\star}$ & Joint replacement \\
\hline Telephone contact & Analgesics & Osteotomy \\
\hline Exercise & Opioids & $\begin{array}{l}\text { Arthroscopic } \\
\text { debridement }\end{array}$ \\
\hline $\begin{array}{l}\text { Footwear, including } \\
\text { insoles }\end{array}$ & SYSADOA* & Lavage \\
\hline Sticks & Psychotropic drugs & \\
\hline Spa & Sex hormones & \\
\hline Patellar tape & $\begin{array}{l}\text { Topical (periarticular) } \\
\text { treatment }\end{array}$ & \\
\hline Diet & Intra-articular steroids & \\
\hline Vitamins & $\begin{array}{l}\text { Intra-articular hyaluronic } \\
\text { acid }\end{array}$ & \\
\hline
\end{tabular}

«NSAIDs = non-steroidal anti-inflammatory drugs; SYSADOA = symptomatic slow acting drugs for osteoarthritis.

European countries, together with two experts in the field of guidelines methodology.

The objectives of the committee were $(a)$ to describe the treatments available for the treatment of knee OA and to review the current level of evidence for their effectiveness and $(b)$ to produce recommendations for the management of knee OA based on a combination of current evidence based and expert opinion.

SCOPE OF THE REVIEW

During the first meeting the committee produced a comprehensive list of treatment modalities to be included in the review process (table 1). To focus the review process towards clinically relevant issues, the committee also agreed 10 clinically important questions related to the management of $\mathrm{OA}$ of the knee.

\section{EVIDENCE BASED REVIEW}

Search strategy

A systematic search was devised and conducted using two databases, Medline Ovid and BIDS Embase. The search strategy was designed to be inclusive. All European language publications in the form of systematic reviews, meta-analyses, randomised controlled trials, controlled trials, and observational studies were included. Publications in non-European languages were excluded. Several search headings were "exploded", including osteoarthritis, randomised controlled trial, meta-analysis, and management. The reference studies discovered in review papers and meta-analyses were also included. The searches were conducted for the period January 1966 to December 1998 and were last performed in July 1999.

\section{Selection of manuscripts}

All trials that assessed the effects of one of the specified treatments on OA of the knee were selected. For the purpose of this review OA of the knee was defined as patients with clinical and/or radiographic evidence of knee OA. Only papers exclusively studying knee OA were included in the quantitative analysis; those studying OA in more than one joint (for example, hip plus knee OA) were excluded after qualitative assessment.

The methodological design of each study was scored according to a predetermined system. ${ }^{3}$ This methodological checklist provided a quality assessment of the reporting, external and internal validity, and the statistical power of each study. Studies were scored $0-1$ for 26 questions and $0-2$ for one question, giving a maximum score of 28 . Power calculations were scored as 1 if present and 0 if absent. English language publications were scored by three assessors. Other European language publications were assessed by individual members of the EULAR Steering Committee
Table 2 Categories of evidence

\begin{tabular}{ll}
\hline Category & Evidence from: \\
\hline 1A & Meta-analysis of randomised controlled trials \\
1B & At least one randomised controlled trial \\
2A & At least one controlled study without randomisation \\
2B & At least one type of quasi-experimental study \\
3 & Descriptive studies, such as comparative studies, correlation \\
& studies, or case-control studies \\
4 & Expert committee reports or opinions and/or clinical experience \\
& of respected authorities \\
\hline
\end{tabular}

Table 3 Strength of recommendation

$\begin{array}{cc}\text { A } & \text { Directly based on category } 1 \text { evidence } \\ \text { B } & \text { Directly based on category } 2 \text { evidence or extrapolated } \\ & \text { recommendation from category } 1 \text { evidence } \\ \text { C } & \text { Directly based on category } 3 \text { evidence or extrapolated } \\ & \text { recommendation from category } 1 \text { or } 2 \text { evidence } \\ \text { D } & \text { Directly based on category } 4 \text { evidence or extrapolated } \\ & \text { recommendation from category } 2 \text { or } 3 \text { evidence }\end{array}$

fluent in the language of the publication. All quality assessments were collected and recorded centrally.

\section{Estimation of treatment effect}

Quantitative analysis of treatment effect was assessed, where possible, by calculating the effect size (ES) ${ }^{4}$ and/or the number needed to treat $(\mathrm{NNT})^{5}$ for validated outcome measures of pain and physical function. Before the calculation of these quantitative measures all studies were assessed for the availability of adequate summary statistics. The mean and distribution of values for the baseline, end point, and difference from baseline to end point were tabulated for each of the outcome measures recorded. The $\mathrm{Z}$ and $\mathrm{T}$ scores and $\mathrm{p}$ values were also recorded when available. Effect size calculations were performed with meta-analysis software. ${ }^{6}$ Pooled variance of baseline values was used as an estimation of the pooled variance of the difference between baseline and end point. The NNT was estimated for $20 \%$ reduction in pain outcome measures from baseline. This calculation was performed on continuous data (visual analogue scale (VAS)) using an assumption that the values for change displayed a normal distribution about the mean. In the absence of data allowing direct quantification of the treatment effect, studies were simply classified as to the presence or absence of a statistically significant improvement in outcome.

\section{Categorising evidence}

Evidence was categorised according to study design reflecting susceptibility to bias. Table 2 shows the categories in descending order of importance. Categories of evidence were adapted from the classification of the United States Agency for Health Care Policy and Research. Questions were answered using the best evidence available. If, for example, a question on the effect of intervention could be answered by category 1 evidence, then studies of a weaker design (controlled trials without randomisation) were not reviewed.

\section{Strength of recommendation}

The strength of recommendation for an intervention was graded A-D (table 3) by the four members of the editing subcommittee of the task force, after examination of the evidence in detail. The strength of recommendations is based not only on the level of evidence but also on consideration of the following: the effect size of the intervention; the side effect profile, the applicability of the evidence to the population of interest, the practicality of delivery; and economic considerations. 


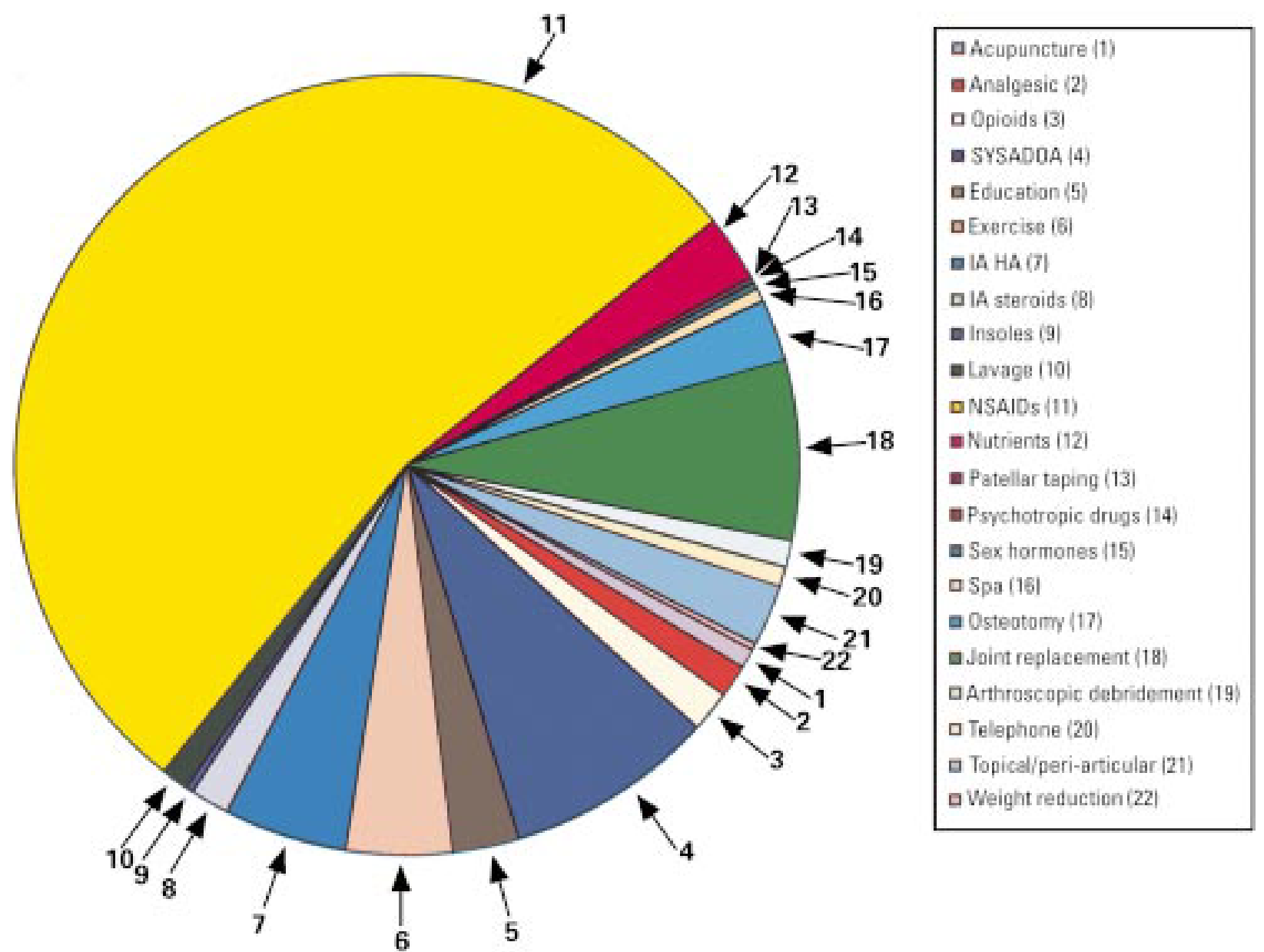

Figure 1 Interventions assessed in clinical trials of knee osteoarthritis. SYSADOA = symptomatic slow acting drugs for osteoarthritis; $I A$ HA = intra-articular hyaluronic acid; NSAID = non-steroidal anti-inflammatory drug.

ASSESSMENT OF EXPERT PANEL OPINION

Experts'opinion approach

After informing the experts about the results of the literature research and the meetings that discussed the main advantages and disadvantages of the different treatment modalities, a questionnaire was sent to all experts asking for their overall personal recommendation for each intervention. This was expressed by a VAS, in which 0 represented the statement "I do not recommend this treatment modality" and 100 represented the statement "I fully recommend this treatment modality". Results are presented as the mean and standard deviation of the scores.

Two sets of recommendations were elaborated: $(a)$ the final expert recommendations on treatment, and (b) recommendations for a research agenda. The experts decided arbitrarily to provide 10 short sentences summarising their opinions for each list. A Delphi approach was then employed to reach a consensus. All the recommendations were collated (121 for the final treatment propositions and 110 for the research agenda) and then sent back to the experts with the request to rank the most important 10 propositions to be included in the final set of recommendations. For the second round of the Delphi exercise, only those recommendations which received approval from at least five experts were retained ( 25 for the final treatment propositions and 24 for the research agenda). The same approach was used for the third round (17 propositions for treatment recommendations and 18 for the research agenda propositions), resulting in eventual determination of the most agreed 10 short sentences in each set.
Level of evidence of the experts'opinion approach

The researchers who undertook the literature research evaluated the level of evidence of each of the 10 final propositions of the experts.

\section{Results}

EVIDENCE BASED APPROACH

Two thousand eight hundred and ninety two studies were identified by the search strategy, 744 were intervention trials involving knee OA. Six hundred and eighty of these trials used at least one of the 23 treatment modalities outlined in table 1. Three hundred and sixty five trials assessed a non-steroidal anti-inflammatory drug (NSAID) as the primary intervention. Table 4 summarises the quality assessment score (QS) and the calculation of the ES for pain reduction for each intervention. From the 680 studies, 61 (31 placebo controlled) provided adequate summary statistics for knee OA to enable the ES for pain reduction to be estimated. Of the 61 studies, 11 provided enough data to allow the calculation of the true ES. A calculation of ES for functional improvement under Lequesne's index was performed on seven studies and is summarised in table 5 .

Only five studies on knee OA alone provided sufficient data to calculate the number needed to treat to gain a $20 \%$ reduction in pain $\left(\mathrm{NNT}_{20}\right)$. Two studies involved topical interventions, one used capsaicin gel $^{7}$ (QS 21) $\mathrm{NNT}_{20} 5.5$ and one used diclofenac $\mathrm{gel}^{8}$ (QS 22) $\mathrm{NNT}_{20}$ 6.8. Two studies involved intra-articular hyaluronic acid, but the trial period varied considerably between the studies. In one study $^{9}$ (QS 22) outcome measures were recorded over 343 days, $\mathrm{NNT}_{20} 40.2$, whereas in another study ${ }^{10}$ (QS 25) over 
Table 4 Summary of the effect sizet (ES) and quality assessment score (QS) for 23 treatment modalities in knee osteoarthritis (OA)

\begin{tabular}{|c|c|c|c|c|c|c|c|c|c|}
\hline Intervention & $\begin{array}{l}\text { Total } O A \\
\text { papers }\end{array}$ & $\begin{array}{l}\text { Positive to } \\
\text { control }\end{array}$ & $\begin{array}{l}\text { ES No of } \\
\text { trials }\end{array}$ & $E S$ (median) & ES (range) & $\begin{array}{l}\text { Quality scores } \\
\text { (median) }\end{array}$ & $Q S$ (range) & $\begin{array}{l}\text { Category of } \\
\text { evidence }\end{array}$ & $\begin{array}{l}\text { Strength of } \\
\text { recommendation }\end{array}$ \\
\hline Analgesic & 10 & 5 & & & & 15.5 & $7-24$ & $1 \mathrm{~B}$ & $\mathrm{~A}$ \\
\hline Opioid & 10 & 6 & & & & 19 & $11-27$ & 1B & B \\
\hline SYSADOA $\ddagger$ & 59 & 26 & 6 & 1.21 & $0.28-1.50$ & 16 & $1-26$ & $1 \mathrm{~B}$ & A \\
\hline Patient education & 19 & 8 & 4 & 0.23 & $0.05-0.35$ & 13 & $7-17$ & $1 \mathrm{~A}$ & A \\
\hline Exercise & 31 & 15 & 4 & 0.78 & $-0.58-1.05$ & 13 & $7-20$ & $1 \mathrm{~B}$ & A \\
\hline IA $\ddagger$ hyaluronic acid & 34 & 21 & 4 & 0.49 & $0.04-0.9$ & 20 & $7-26$ & 1B & B \\
\hline IA steroids & 10 & 5 & 1 & 1.27 & & 16 & $4-22$ & 1B & A \\
\hline Insoles & 3 & 2 & & & & 10 & $3-11$ & $2 \mathrm{~A}$ & $\mathrm{~B}$ \\
\hline Lavage & 6 & 3 & 1 & 0.84 & & 13.5 & $11-18$ & 1B & B \\
\hline NSAID $\ddagger$ & 365 & 214 & 4 & 0.49 & $0.16-1.19$ & 16 & $2-27$ & $1 \mathrm{~A}$ & $\mathrm{~A}$ \\
\hline${ }^{\star}$ Nutrients & 19 & 15 & 1 & 0.65 & & 17 & $4-27$ & $2 \mathrm{~A}$ & C \\
\hline Patellar taping & 1 & 1 & & & & 15 & & $1 \mathrm{~B}$ & B \\
\hline Psychotropic drugs & 2 & 1 & & & & 19 & $16-22$ & $1 \mathrm{~B}$ & $\mathrm{~B}$ \\
\hline Sex hormones & 1 & & & & & 15 & & $2 \mathrm{~B}$ & $\mathrm{C}$ \\
\hline Spa & 4 & 3 & 1 & 0.47 & & 14.5 & $12-17$ & $1 \mathrm{~B}$ & $\mathrm{C}$ \\
\hline Osteotomy & 17 & & & & & 11 & $7-15$ & 3 & $\mathrm{C}$ \\
\hline Joint replacement & 51 & 1 & & & & 12 & $4-23$ & 3 & $\mathrm{C}$ \\
\hline $\begin{array}{l}\text { Arthroscopic } \\
\text { debridement }\end{array}$ & 8 & 1 & & & & 10 & $2-17$ & $1 \mathrm{~B}$ & $\mathrm{C}$ \\
\hline Telephone contact & 5 & 3 & 1 & 1.09 & & 18 & $16-18$ & $1 \mathrm{~B}$ & $\mathrm{~B}$ \\
\hline Topical/periarticular & 18 & 11 & 3 & 0.56 & $-0.05-0.91$ & 19 & $9-26$ & $1 \mathrm{~B}$ & A \\
\hline Weight reduction & 1 & & & & & 13 & & 1B & B \\
\hline
\end{tabular}

^Nutrients include trial dietary supplements, vitamins, and minerals.

†Effect size recorded for intervention against placebo.

¥SYSADOA = symptomatic slow acting drugs for osteoarthritis; NSAIDs = non-steroidal anti-inflammatory drugs; IA = intra-articular.

35 days the $\mathrm{NNT}_{20}$ was 7.2. Only one NSAID study, on oral diflunisal $^{11}$ (QS 16), suggested that 5.9 patients need to be treated to gain a $20 \%$ reduction in pain over a 42 day period.

Of the 23 modalities, 16 were supported by evidence from at least one randomised controlled trial and were graded as either 1A or 1B for category of evidence. From surgical studies only those concerned with arthroscopic debridement and patellar replacement were supported by evidence from randomised controlled trials. Osteotomy and total joint replacement were only supported by category 3 evidence. Only seven of the 16 modalities supported by grade 1 evidence received an " $A$ " for strength of recommendations from the panel of experts (table 4).

\section{EXPERTS' OPINION APPROACH}

Figure 2 summarises the overall opinion of the experts of the usefulness of each treatment modality. The experts gave similar weighting to oral drugs such as paracetamol and NSAIDs, intra-articular treatments such as steroids, non-pharmacological treatments such as patient education and exercise, and surgical treatment such as joint replacement. Tables 6 and 7 summarise the final treatment recommendations and the research agenda.

ASSESSMENT OF PROPOSITIONS

These propositions are in no ranked order.

Treatment of knee $O A$ should be tailored to the individual patient, taking into account factors such as age, comorbidity, and the presence of inflammation

This statement represents ideal practice and the decisionmaking that is required in clinical practice. Clinical trials predominantly investigate the efficacy of one or two specific single treatments in highly selected homogeneous groups of otherwise fit subjects with knee OA. Derived data are therefore not directly applicable to the whole population of subjects with OA. Furthermore, few studies have examined the predictors of response to treatment required to produce the evidence base to allow such decisions to be made. As well as the expected relative benefits and potential dangers of the interventions themselves, other aspects specific to the patient must clearly be taken into account. This has relevance to both medical and surgical interventions. The holistic approach to the patient is universally accepted. It has obvious face validity but no research based justification specific to knee OA.

Optimal management of knee $O A$ requires a combination of pharmacological and non-pharmacological treatment modalities

Few studies have directly considered this issue using appropriate factorial randomised controlled trials. Ravaud et al performed a factorially designed randomised controlled trial of 98 patients with knee OA (QS 18), assessing the use of joint lavage plus intra-articular steroid over either treatment alone. ${ }^{12}$ The results suggested additional benefit, most marked in the first month, of combining the two modalities. A non-randomised, controlled study of 107 patients (QS 11), showed a significant improvement in pain reduction using wedged insoles and oral indometacin in combination compared with indometacin alone (ES for pain $=0.5) .{ }^{13}$

There is indirect evidence that non-pharmacological modalities offer additional benefit over and above analgesic use from randomised controlled trials in which all subjects were receiving analgesics at baseline. These have shown that a home exercise programme ${ }^{14}$ (QS 13), physiotherapy $^{15}$ (QS 19), and education ${ }^{16}{ }^{17}$ (QS 12, 17) offer additional benefit when used in addition to an analgesic regimen.

Table 5 Effect size (ES) of intervention on functional outcome in knee osteoarthritis

\begin{tabular}{|c|c|c|c|c|c|c|c|c|c|}
\hline Intervention & $\begin{array}{l}\text { No of } \\
\text { patients }\end{array}$ & Withdrawals & Control & $\begin{array}{l}\text { Study length } \\
\text { (days) }\end{array}$ & $\begin{array}{l}\text { Functional } \\
\text { outcome }\end{array}$ & $E S$ & Blinding & $\begin{array}{l}\text { Randomisati } \\
\text { on }\end{array}$ & $Q S$ \\
\hline Aceclofenac & 335 & 99 & Diclofenac & 182 & Lequesne & 0.04 & Double & Yes & 26 \\
\hline Chondroitin sulphate & 146 & 20 & Diclofenac & 180 & Lequesne & 0.88 & Double & Yes & 20 \\
\hline Chondroitin sulphate & 85 & 5 & Placebo & 182 & Lequesne & 1.01 & Double & Yes & 24 \\
\hline Chondroitin sulphate & 127 & 6 & Placebo & 91 & Lequesne & 1.32 & Double & Yes & 21 \\
\hline Diclofenac & 155 & 13 & Placebo & 15 & Lequesne & 0.62 & Double & Yes & 22 \\
\hline GAGPS ${ }^{\star}+$ (Arteparon) & 80 & 9 & Placebo & 182 & Lequesne & 0.11 & Double & Yes & 21 \\
\hline Hyaluronic acid & 110 & 22 & Placebo & $343(28)$ & Lequesne & $0.48(0.42)$ & None & Yes & 22 \\
\hline
\end{tabular}

${ }^{\star}$ Currently unavailable in Europe.

GAGPS = glycosaminoglycan polysulphuric acid 


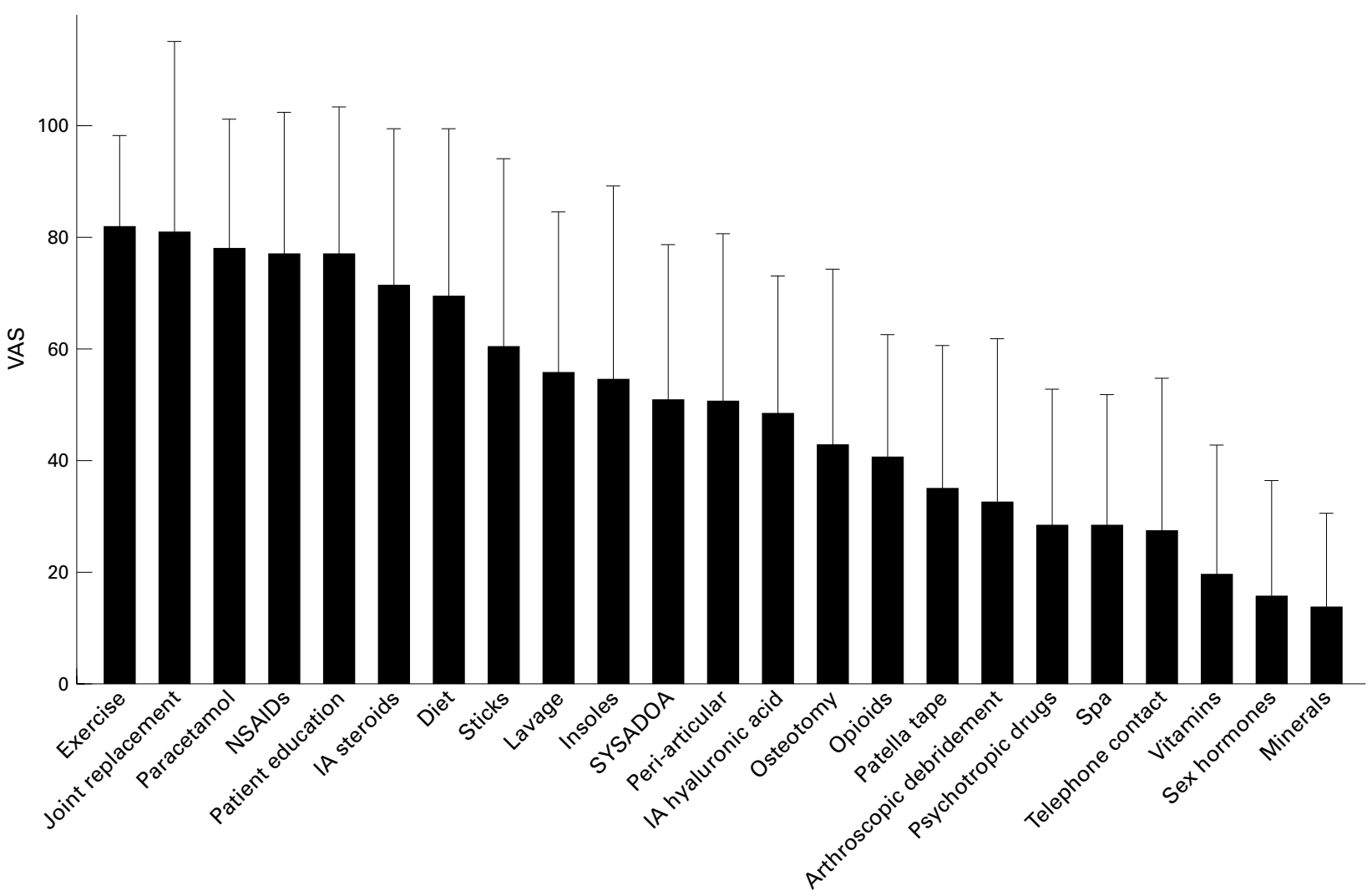

Figure 2 Overall experts'opinion of the usefulness of the different treatment modalities. Results are given as the means (SD) of the opinions of the 21 experts. VAS = visual analogue scale, in which $0=I$ do not recommend this treatment modality and $100=I$ strongly recommend this treatment modality; NSAIDs = non-steroidal anti-inflammatory drugs; SYSADOA = symptomatic slow acting drugs for osteoarthritis; $I A=$ intra-articular.

There is therefore a reasonable evidence base to support this statement $(1 \mathrm{~B})$.

Paracetamol is the oral analgesic to try first and, if successful, is the preferred long term oral analgesic

Paracetamol is commonly used as self medication for the treatment of mild to moderate pain. It is the recommended initial oral analgesic for knee OA in published guidelines. ${ }^{12}$ However, few studies have directly assessed the efficacy of paracetamol in knee OA. One six week randomised controlled trial in just 25 patients $^{18}$ (QS 21) showed a significant improvement in pain at rest from paracetamol compared with placebo. Two studies comparing paracetamol with an oral NSAID in knee OA recorded within-

Table 6 Propositions of final recommendations based on the opinion of the experts

Propositions

Treatment of knee $\mathrm{OA}^{\star}$ should be tailored to the individual patient, taking into account factors such as age, comorbidity, and the presence of inflammation

Optimal management of knee OA requires a combination of pharmacological and non-pharmacological treatment modalities

Intra-articular injection of long acting steroid is indicated for acute exacerbation of knee pain, especially if accompanied by effusion

There is evidence that SYSADOA* (glucosamine sulphate, chondroitin sulphate, diacerein, and hyaluronic acid) may possess structure modification properties, but more studies, using standardised methodology are required

Hyaluronic acid and other SYSADOA are probably effective in knee OA, but the size effect is relatively small, suitable patients are not well defined, and pharmacoeconomic aspects of that treatment are not well established

Non-pharmacological treatment of knee OA should include regular education, exercise, appliances (sticks, insoles), and weight reduction

Exercises, especially those directed towards increasing strength of quadriceps and/or preserving normal mobility of the knee are strongly recommended

Paracetamol is the oral analgesic to try first and, if successful, is the preferred long term oral analgesic

NSAIDs ${ }^{\star}$ (oral or topical) should be considered in patients (with effusion) unresponsive to paracetamol

10 Joint replacement has to be considered for refractory pain associated with disability and radiological deterioration

${ }^{\star} \mathrm{OA}=$ osteoarthritis; NSAIDs = non-steroidal anti-inflammatory drugs; SYSADOA = symptomatic slow acting drugs for osteoarthritis.

Table 7 Propositions to be included in the research agenda based on the opinion of the experts

Propositions

There is a need to establish a set of recommendations for uniform and full reporting of clinical trials in knee $\mathrm{OA}^{\star}$

There is an urgent need to examine the efficacy and cost utility of surgical techniques

The indications for joint replacement need to be determined

Studies are still required to determine the most efficient effective home exercises for knee OA

The clinical relevance of any structural effect of SYSADOA* requires evaluation

What are the long term effects of COX inhibition on joint tissues, primarily, cartilage and bone?

Studies should include quality of life and function, as well as pain, as outcome measures

Clinical predictors of response to pharmacological and non-pharmacological interventions need to be determined

Randomised controlled trials should more fully assess the various non-pharmacological interventions for knee OA

COX-2 specific inhibitors should be compared with paracetamol and with low doses of conventional NSAIDs in patients with knee OA who have chronic pain

${ }^{\star} \mathrm{OA}=$ osteoarthritis; NSAIDs = non-steroidal anti-inflammatory drugs; SYSADOA = symptomatic slow acting drugs for osteoarthritis. 
group reduction in pain with paracetamol. ${ }^{19}{ }^{20}$ One four week randomised controlled trial showed that paracetamol $4 \mathrm{~g}$ /day was as effective as ibuprofen (up to $2400 \mathrm{mg} /$ day) ${ }^{19}$ (QS 21). Another randomised controlled trial showed that paracetamol can be used effectively in doses of up to $2400 \mathrm{mg}$ over 2 years without significant adverse outcomes $^{20}$ (QS 23). There are no drug interactions or common contraindications to the use of paracetamol, including its use in the elderly.

In summary, there is evidence (1B) that paracetamol is effective in the treatment of knee OA and that in many patients it is comparable with ibuprofen in the short term. There is also evidence (1B) that paracetamol may be taken safely over the long term. Clearly, a drug that is both safe and commonly effective should be considered early in the management of knee OA and, if effective, as an integral component of long term pain control.

\section{NSAIDs (oral or topical) should be considered in patients} (with effusion) unresponsive to paracetamol

With the increasing focus on the low grade inflammatory component of OA, NSAIDs would appear to be logical drugs in patients unresponsive to paracetamol, particularly in the presence of clinically overt synovitis. There is, however, no direct evidence base to support this statement. Numerous studies have shown that oral NSAIDs are better than placebo (ES median 0.49, range 0.16-1.19), confirming the efficacy of NSAIDs in the management of knee OA.

A few trials have directly compared paracetamol and NSAIDs. They have generally, but not exclusively, found NSAIDs to have better efficacy than paracetamol but with increased gastrointestinal side effects. In a two year randomised controlled trial ${ }^{20}$ (QS 23) paracetamol was compared with naproxen in 178 patients. Naproxen led to greater reductions in pain than paracetamol (ES 0.32 after 42 days and 0.45 after 730 days). Patient drop out was high $(65 \%)$, owing to lack of efficacy in the paracetamol arm and adverse events in the naproxen arm. A four week randomised controlled trial comparing paracetamol and ibuprofen in patients with knee OA, with signs of joint inflammation, failed to show any significant benefit of ibuprofen over paracetamol. ${ }^{19}$

Topical NSAIDs are a useful option in those unwilling or unable to take oral NSAIDs. A randomised controlled trial of topical diclofenac including 155 patients $^{8}$ (QS 22) recorded a positive ES of 0.91 compared with placebo. Another study with eltenac involving 281 patients $^{21}$ (QS 26) recorded a negative ES -0.05 , but suggested benefit in those with severe disease.

There is good evidence (1B) for the efficacy of NSAIDs, both oral and topical, in the management of knee OA. There is good evidence that they are more efficacious than paracetamol but the statement that they should be used in patients in whom paracetamol has failed, though attractive, does not have an evidence base to support it.

Intra-articular injection of long acting steroid is indicated for acute exacerbation of knee pain, especially if accompanied by effusion

The effects of steroids in knee OA have been assessed in a number of studies. One randomised controlled trial concluded that steroid was more effective than placebo for pain relief over seven days (ES 1.27) in patients with knee OA, not all of whom had effusions ${ }^{22}$ (QS 19). Another randomised controlled trial involving 98 patients showed a significant difference between intra-articular steroid and placebo after one week but no difference after 24 weeks, supporting only relatively short term benefit from this treatment $^{12}$ (QS 18).
Few studies have assessed predictors of response. One randomised controlled trial involving 84 patients confirmed short term improvement of symptoms for steroid over placebo and found a better outcome in those with an effusion $^{23}$ (QS 22). However, a randomised crossover study of methylprednisolone $v$ saline found no clinical predictors of response, suggesting that steroid injection should not be reserved just for those with effusion ${ }^{24}$ (QS 17).

In conclusion, there is evidence $(1 \mathrm{~B})$ that intra-articular steroids are efficacious but their benefit may be relatively short lived. The evidence for predictors of response, however, remains unclear, and further studies are needed to consider this question.

There is evidence that symptomatic slow acting drugs for $O A$ (SYSADOA) (glucosamine sulphate, chondroitin sulphate, diacerein, and hyaluronic acid) may possess structure modification properties, but more studies, using standardised methodology are required

Promising data exist from animal models of OA, but there are few clinical trials in humans. A 12 month randomised controlled trial of 39 patients with knee OA showed that intra-articular hyaluronan delayed the structural progression of $\mathrm{OA}$ as assessed by arthroscopic assessment of articular cartilage. ${ }^{25}$ More studies using standardised methodology are required before we can propose a disease modifying role for these compounds.

Hyaluronic acid and other SYSADOA are probably effective in knee $O A$, but the size effect is relatively small, suitable patients are not well defined, and pharmacoeconomic aspects of that treatment are not well established

The introduction of hyaluronic acid has been viewed as an advance in the management of OA. Its role in pain reduction, functional improvement, and disease modification in knee OA has been assessed. Until 1998, 32 trials assessed the efficacy of hyaluronic acid in relation to the knee joint. Three randomised controlled trials that allowed calculation of effect size recorded significant reductions in pain against placebo (ES $0.04,0.49,0.9$ ) over time periods of 60 days to one year ${ }^{9627}$ (QS 22, 19, 14). One study recorded functional improvements on Lequesne's index (ES 0.36) over one year. ${ }^{9}$ One study looked at endoscopic changes over the short term and showed a decrease in disease progression over one year. ${ }^{25}$ However, no studies considered possible predictors of response, and no studies until the end of 1998 provided evidence for a role in disease modification in the long term.

The use of SYSADOA in the management of knee OA varies widely across Europe. Such variations in practice depend on a number of factors, from local availability of the product to the lack of guidelines to a doctor's personal choice. On reviewing the literature 59 studies were identified involving SYSADOA in knee OA. The products were assessed individually. For chondroitin sulphate, three randomised controlled trials recorded a significant improvement in knee pain over placebo (ES 1.23, 1.5, 1.37) ${ }^{28-30}$ (QS 24, 25, 21), two of which recorded similar improvements in function (ES 1.01, 1.32). ${ }^{29} 30$ Such improvements were also recorded in a six month randomised controlled trial, following direct comparison with diclofenac (ES 1.44) $)^{31}$ (QS 20).

Three studies involved glucosamine sulphate. Compared with placebo, glucosamine appears to be effective for both pain reduction (ES 1.02) as recorded in one controlled study over eight weeks ${ }^{32}$ (QS 14) and for functional improvement as recorded in one randomised controlled trial $^{33}$ (QS 26). When compared directly with an NSAID (ibuprofen) the effect on pain was comparable over four weeks $^{34}$ (QS 23). 
Twenty nine studies assessed the efficacy of glycosaminoglycan polysulphuric acid (GAGPS), though many were of poor quality and few focused on knee OA alone. Two randomised controlled trials recorded improvements in knee pain when compared with placebo (ES 1.18, 0.28) ) $^{35}{ }^{36}$ (QS $18,21)$. One of these studies also recorded improvements in function (ES 0.11) over six months. ${ }^{36}$ However, GAGPS (Arteparon) is currently unavailable in Europe.

In summary, there is evidence to support the efficacy of hyaluronic acid in the management of knee OA both for pain reduction (1B) and functional improvement (1B). However, although pain relief may be obtained for several months, rather than for several weeks as with steroid, this benefit may be offset by its slower onset of action and by the requirement of a course of three to five injections a week with the logistical and cost issues that entails. There is no evidence for a role in disease modification. The term SYSADOA covers a range of agents and although, again, there is some evidence to support the use of two of these agents, glucosamine sulphate (1B) and chondroitin sulphate (1B), the evidence for others is weak or absent.

\section{Non-pharmacological treatment of knee $O A$ should include regular education, exercise, appliances (sticks, insoles), and weight reduction}

Education should form an integral part in the management of any chronic disease. Practitioners should always explain to the patient the nature of their condition, its prognosis, the requirement of investigations and what that involves, and determine with the patient the rationale and practicalities of their individualised management plan. However, in addition to professional responsibility, education itself may have an impact on the outcome of disease. Several large randomised controlled trials and a metaanalysis have shown benefits of different educational techniques in reducing pain and increasing coping skills, but with little impact on function in patients with knee OA. ${ }^{37}$ Education techniques shown to be effective include individualised education packages ${ }^{16}$ (QS 12), regular telephone calls ${ }^{17}$ (QS 17), patient coping skills, ${ }^{38}{ }^{39}$ (QS 15, 13) and spouse-assisted coping skills training ${ }^{40}$ (QS 15).

Exercise can be divided into joint-specific strength and range of motion exercises and general aerobic conditioning. There is evidence from large randomised controlled trials that joint-specific exercises reduce pain and improve function in patients with knee $\mathrm{OA}^{1415}$ (QS 19, 13), as do aerobic exercise regimens ${ }^{15}{ }^{41}$ (QS 19,20). The median effect size for exercise is 0.78 (range -0.58-1.05). Importantly, some of these studies report long term improvements (6-18 months)

No randomised controlled trials have considered the use of insoles or walking sticks in the management of knee OA. Two controlled studies of insoles ${ }^{1342}$ (QS 11, 10), however, both showed an improvement over the control.

Although weight reduction is recommended to virtually all patients with knee OA, the relation between weight reduction and knee OA has only been assessed formally in two studies. A randomised placebo controlled trial on the use of phentermine in generalised $\mathrm{OA}^{43}$ (QS 13) showed an improvement in pain compared with baseline but not compared with placebo. A large cohort study ${ }^{44}$ (QS 15) showed that weight loss reduced the risk of developing symptomatic knee OA in women.

There is therefore good evidence that education (1A) and exercise regimens $(1 \mathrm{~B})$ reduce pain in knee $\mathrm{OA}$ and that exercise regimens also improve function. The use of appliances and advising weight reduction both seem sensible options in patients with knee OA, but are only supported by relatively weak evidence at present.
Exercises, especially those directed towards increasing strength of quadriceps and/or preserving normal mobility of the knee are strongly recommended

Referral to a physiotherapist is one of the mainstays of treatment for patients with knee OA. The efficacy of quadriceps strengthening exercises in patients with knee OA has been examined in nine studies. All studies recorded improvements in pain when compared with baseline values. Four randomised controlled trials ${ }^{14} 154546$ (QS 19, $14,13,18$ ) were identified including a total of 531 patients. All trials recorded significant improvements in pain when compared with the control groups (ES 1.05). ${ }^{45}$ Two of the studies $^{14}{ }^{15}$ also showed a significant improvement in functional index compared with the control group. Such exercise need not necessarily require regular professional supervision. ${ }^{14}$ Interestingly, when strengthening exercise was directly compared with aerobic fitness training for knee $\mathrm{OA}^{15}$ both seemed equally efficacious over 18 months. Many regimens combine elements of both forms of exercise, though whether the effects of both are additive remains uncertain.

There is therefore good evidence (1B) that quadriceps strengthening exercises are beneficial in the management of knee OA. There is also evidence, however, that fitness training has long term benefits for pain and function from knee OA.

\section{Foint replacement has to be considered for refractory pain} associated with disability and radiological deterioration

There is strong clinical agreement that patients severely incapacitated by OA can have their lives transformed by knee replacement surgery. Difficulty arises when an attempt is made to assess the strength of evidence for a given surgical approach or an attempt made to compare this approach with other treatment modalities. Of 51 studies identified in this area, no studies compared surgery with non-surgical management. Twenty eight studies focused on total knee replacement, 17 on unicompartmental knee replacement, while six focused on patellofemoral joint resurfacing or replacement. All studies reported improvements in pain and/or function compared with baseline parameters. Although it is acknowledged that difficulties with study design may limit randomised studies on surgical treatments, there are areas which should be explored, including predictors of response, differences in surgical technique, or joint prosthesis on long term outcomes.

The influence on long term outcome of knee replacement, expressed in terms of "implant survival", of various factors (patient, procedure, implant, and surgeon related) have been examined using the Swedish nationwide knee replacement registries. ${ }^{47}$ Although a strength of these studies is their power (based on large numbers), generalisability, and long term follow up, a limitation is created by their use until recently of implant revision only as the outcome measure. The relation between implant revision and patient satisfaction or other outcomes relevant to the patient is uncertain.

\section{Discussion}

These are the first clinical guidelines on knee OA to be developed by EULAR. As defined by the Institute of Medicine clinical guidelines are "Systematically developed statements to assist practitioner and patient decisions about appropriate health care for specific clinical conditions". ${ }^{48}$ An evidence based approach was specifically chosen by the task force to minimise the potential limitations of "expert opinion" alone ${ }^{49}$ and to permit, wherever possible, calculation of effect sizes and NNT to allow comparison of efficacy between diverse forms of treatment. Importantly, the type of evidence to substantiate the statements 
made (ranging from robust research evidence to expert opinion alone) is explicitly stated.

Despite the strengths of this method of guideline development a number of caveats deserve emphasis. Although we employed a traditional search strategy, used two recognised large computerised databases, and extended the search to include non-English languages represented on the task force, some study data might have been excluded from our review. Furthermore, our interest was restricted to the knee and we did not include studies for the calculation of effect size or NNT having mixed patient groups with OA where summary statistics were not available for the knee alone. Importantly, we had to set a finite end date for the search (December 1998) and restricted our deliberations to the evidence that arose from that search. We recognise, of course, that other studies have been reported during the interval to publication and that the guidelines will require regular update and review in the light of new evidence and evolving methods of healthcare delivery.

A further issue is the comparability and quality of the research evidence. Different studies varied greatly in their design-for example in the type of patient with OA studied, the availability of concomitant analgesic drugs, the outcome measures used, and the duration of treatment, all of which hamper determination of comparative efficacy. Furthermore, the quality of the studies varied. We used a recognised quality assessment measure that is suited for a variety of study designs. "Quality" scores, however, assess not only the design and rigour of a study but also the presentation of the information. ${ }^{50}$ We therefore did not exclude studies purely because of low total scores. However, it was noteworthy that the scores obtained were not high and that few studies provided sufficient information to calculate effect size or NNT. Interestingly, there was little difference in quality scores between the different treatment modalities, the lowest mean scores occurring in nonpharmacological studies with their inherently greater difficulties of blinding and randomised controlled trial design.

It was of interest that there was often discordance between the research evidence and the opinion of the experts. It would seem that much of our practice is governed more by our clinical experience, local situation, and individual bias than the balance of published evidence. This was even the more striking with such broad representation from across Europe. Varying attitudes towards the nature of knee $\mathrm{OA}$ in the countries represented, varying access to health professionals, different healthcare delivery systems, and different methods of funding all probably contributed to discrepancies between experts in their weighting of individual treatment strategies. Nevertheless, despite such differences the Delphi system permitted consensus agreement on the issues presented.

It is apparent that there are important areas of clinical practice for which there is no clear evidence based research. There was a general paucity of well designed randomised controlled trials to consider the clinical questions raised. In particular, few studies examined long term treatment outcome (12 months or longer), even though knee $\mathrm{OA}$ is associated with chronic or intermittent symptoms that spread over many years. Also, few studies sought clinical predictors of treatment response, such as $x$ ray severity, presence of clinical inflammation, age, or obesity. Unfortunately, restricting patient entry to ensure homogeneity for clinical variables excludes determination of such predictors and greatly limits the generalisability of the study findings. ${ }^{51}$ There are therefore few data to guide treatment choice for long term management of heterogeneous patients with "knee OA". An important function of these guidelines has been to emphasise such gaps in our knowl- edge and to help inform the future research agenda on clinically relevant issues.

The task force made no attempt to produce stepwise algorithms or to give dogmatic directives for treatment strategies. Any management plan needs to be individualised to take into account holistic factors, such as patient attitudes and knowledge, constitutional features (for example, obesity, muscle weakness, non-restorative sleep), risk factors for OA progression, degree of structural damage, comorbid disease and treatment, treatment availability, and costs. Management also includes several concurrent elements given as a package rather than as sequential single treatments. The task force therefore focused on specific clinical questions and determined, wherever possible, comparative effect sizes for different treatment options. Our findings are in general accord with other published guidelines but present more clearly the evidence on which any statements are based.

The content and conclusions of this document now need to be considered and discussed as widely as possible by healthcare professionals participating in the management of subjects with knee OA. The views of patients will also be sought. EULAR has undertaken to commission regular review of the guidelines to take into account the perspectives of all interested parties, new research evidence, and changes in healthcare delivery. The task force would welcome discussion of this important clinical topic. Contact is invited with any member of the task force.

The authors thank UPSA representatives (Manuela Le Bars and Eric Boccard) for their contributions and UPSA for financial support to meeting costs.

\section{Members of task force}

Maxime Dougados, chairperson, Service de Rhumatologie B, Hôpital Cochin, 27 rue du Faubourg Saint Jacques, 75014 Paris, France Michael Doherty, co-chairperson, Academic Rheumatology, University of Nottingham, Clinical Sciences Building, City Hospital, Hucknall Road, Nottingham NG5 $1 \mathrm{~PB}$, UK

Adrian Pendleton, task force research fellow, Musgrave Park Hospital, Stockmans Lane, Belfast, Ireland

Nigel Arden, task force research fellow, MRC Epidemiology Unit Southampton General Hospital, Tremona Road, Southampton SO16 6YD, UK

Bernard Bannwarth, Laboratoire de Thérapeutique, Université Victor Segalen Bordeaux II 146 rue Léo Saignat, Zone Nord, Bât 1a, BP38 33076 Bordeaux Cedex, France

Johannes WJ Bijlsma, Afdeling Reumatologie en Klinische Immunologie, Academisch ziekenhuis Utrecht, Huispost F02.127 B, Postbus 85500, 3508 GA Utrecht, The Netherlands

Francois Cluzeau, Health Care Evaluation Unit, St George's Hospital Medical School, London SW17 0RE, UK

Cyrus Cooper, MRC Epidemiology Unit, Southampton General Hospital, Tremona Road, Southampton SO16 6YD, UK

Paul A Dieppe, MRC Health Services Research, University of Bristol, Canynge Hall Whiteladies Road, Bristol BS8 2PR, UK

Klaus-Peter Günther, Ortho Klinik/RKU, Oberes Eselsberg 45, D-89081 ULM, Germany

Hans J Hauselmann, Centre for Rheumatology and Bone, Klinik Im Park, Hirslandengruppe, Bellariestrasse 38, CH-8038 Zurich, Switzerland

Gabriel Herrero-Beaumont, Service de Rhumatologie, Hôpital de la Conception, Avenida Reyes Catolicos n², 28040 Madrid, Spain

Phaedon M Kaklamanis, Internal Medicine, Rheumatology, 61 Ipsilantou, 11521 Athens, Greece

Burkhard Leeb, Lower Austrian Centre for Rheumatology, Stockerau Hospital, Landstraße 18, A-2000 Stockerau, Austria

Michel Lequesne, 31-33 rue Guilleminot, 75014 Paris, France

Stefan Lohmander, Department of Orthopaedics, University Hospital, S-22185 Lund, Sweden

Bernard Mazieres, Service de Rhumatologie, CHU Rangueil, Avenue Jean Poulhes, 31054 Toulouse, France

Emilio-Martin Mola, Servicio di Reumatologia, Hospital La Paz, Paseo de la Castellana 261, 28046 Madrid, Spain

Karel Pavelka, Rheumatology Institute, Na Slupi 4, 12850 Praha 2, Czech Republic

Umberto Serni, Divisione di Reumatologia dell' Istituto Ortopedico Toscano, Ortopedico Toscano, Viale Michalangelo 41, Florence, Italy 
Bernd Swoboda, Orthopädische Universitätsklinik und Poliklinik, Waldkrankenhaus St Marian Abteilung fürRheuhopädische Rheumatologie, Rathsberger Straße 57, 91054 Erlangen, Germany

August A Verbruggen, Rheumatology Unit, Universiteit Zuikenhuis, de Pintelaan 185, 9000 Gent, Belgium

Gerd Weseloh, Orthopädische Universitätsklinik und Poliklinik, Waldkrankenhaus St Marian Abteilung fürRheuhopädische Rheumatologie, Rathsberger Strasse 57, 91054 Erlangen, Germany

Irena Zimmermann-Gorska, Department of Rheumatology an Rehabilitation, Karol Marcinskowski University of Medical Sciences in Poznan, 28 Czerwca 1956 r 135/147, 61545 Poznan, Poland

Correspondence to: Professor M Dougados, René Descartes University, Hopital Cochin, Institut de Rhumatologie, Hardy B, 27 rue du Faubourg Saint Jacques, 75014 Paris, France maxime.dougados@cch.ap-hop-paris.fr

1 Hochberg MC, Altman RD, Brandt KD, Clark BM, Dieppe PA, Griffin MR, et al. Guidelines for the medical management of osteoarthritis. Part II.
Osteoarthritis of the knee. American College of Rheumatology [see comments]. Arthritis Rheum 1995;38:1541-6.

2 Scott DL. Guidelines for the diagnosis, investigation and management of osteoarthritis of the hip and knee. Report of a Joint Working Group of the British Society for Rheumatology and the Research Unit of the Royal College of Physicians. J R Coll Physicians Lond 1993;27:391-6.

3 Downs SH, Black N. The feasibility of creating a checklist for the assessment of the methodological quality both of randomized and non-randomized of the methodological quality both of randomized and non-randomized
studies of health care interventions. J Epidemiol Community Health 1998; 52:377-84.

4 Cohen J. Statistical power analysis for the behavioural sciences. New York: Academic Press, 1977.

5 Laupacis A, Sackett DL, Roberts RS. An assessment of clinically useful measures of the consequences of treatment. N Engl J Med 1988;318:172833.

6 Schwarzer R. Meta-Analysis Programs version 5.0. Berlin, Germany: Ralf Schwarzer Computer Programs fo Meta-Analysis, 2000. (www.fuberlin.de/gesund/meta_e.htm)

7 Deal CL, Schnitzer TJ, Lipstein E, Seibold JR, Stevens RM, Levy MD, et al. Treatment of arthritis with topical capsaicin: a double-blind trial. Clin Ther 1991;13:383-95.

8 Dreiser RL, Tisne-Camus M. DHEP plasters as a topical treatment of knee osteoarthritis: a double-blind placebo-controlled study. Drugs Exp Clin Res 1993;19:117-23.

9 Dougados M, Nguyen M, Listrat V, Amor B. High molecular weight sodium hyaluronate (hyalectin) in osteoarthritis of the knee: a 1 year placebocontrolled trial. Osteoarthritis Cartilage 1993;1:97-103.

10 Kolarz G, Kotz R, Broll H, Dunky A, Landsiedl F, Mayrhofer F, et al. Hyaluronic acid in the treatment of osteoarthritis of the knee joint: interim results of a comparative clinical study. Eur J Rheumatol Inflamm 1995;15: results of

11 Lee P, Davis P, Prat A. The efficacy of diflunisal in osteoarthritis of the knee. A Canadian Multicenter Study. J Rheumatol 1985;12:544-8.

12 Ravaud P, Moulinier L, Giraudeau B, Ayral X, Guerin C, Noel E, et al. Effects of joint lavage and steroid injection in patients with osteoarthritis of the knee: results of a multicenter, randomized, controlled trial. Arthritis Rheum 1999;42:475-82.

13 Sasaki T, Yasuda K. Clinical evaluation of the treatment of osteoarthritic knees using a newly designed wedged insole. Clin Orthop Relat Res 1987: 181-7.

14 O'Reilly SC, Muir KR, Doherty M. Effectiveness of home exercise on pain and disability from osteoarthritis of the knee: a randomised controlled trial. Ann Rheum Dis 1999;58:15-19.

15 Ettinger WH Jr, Burns R, Messier SP, Applegate W, Rejeski WJ, Morgan T, et al. A randomized trial comparing aerobic exercise and resistance exercise with a health education program in older adults with knee osteoarthritis. The Fitness Arthritis and Seniors Trial (FAST) [see comments]. JAMA 1997;277:25-31.

16 Mazzuca SA, Brandt KD, Katz BP, Chambers M, Byrd D, Hanna M. Effects of self-care education on the health status of inner-city patients with osteoarthritis of the knee [see comments]. Arthritis Rheum 1997;40:1466-74

17 Weinberger M, Tierney WM, Booher P, Katz BP. Can the provision of information to patients with osteoarthritis improve functional status? A randomized, controlled trial. Arthritis Rheum 1989;32:1577-83.

18 Amadio P, Cummings DM. The evaluation of acetaminophen in the management of osteoarthritis of the knee. Current Therapeutic Research 1983;34:59-66.

19 Bradley JD, Brandt KD, Katz BP, Kalasinski LA, Ryan SI. Treatment of knee osteoarthritis: relationship of clinical features of joint inflammation to the response to a nonsteroidal antiinflammatory drug or pure analgesic. J Rheumatol 1992;19:1950-4.

20 Williams HJ, Ward JR, Egger MJ, Neuner R, Brooks RH, Clegg DO, et al. Comparison of naproxen and acetaminophen in a two-year study of treatment of osteoarthritis of the knee [see comments]. Arthritis Rheum 1993; 36:1196-206.

21 Sandelin J, Harilainen A, Crone H, Hamberg P, Forsskahl B, Tamelander G. Local NSAID gel (eltenac) in the treatment of osteoarthritis of the knee. A double blind study comparing eltenac with oral diclofenac and placebo gel. Scand J Rheumatol 1997;26:287-92.
22 Dieppe PA, Sathapatayavongs B, Jones HE, Bacon PA, Ring EF. Intra-articular steroids in osteoarthritis. Rheumatol Rehabil 1980;19:21217.

23 Gaffney K, Ledingham J, Perry JD. Intra-articular triamcinolone hexacetonide in knee osteoarthritis: factors influencing the clinical response. Ann Rheum Dis 1995;54:379-81

24 Jones A, Doherty M. Intra-articular corticosteroids are effective in osteoarthritis but there are no clinical predictors of response. Ann Rheum Dis 1996;55:829-32

25 Listrat V, Ayral X, Patarnello F, Bonvarlet JP, Simonnet J, Amor B, et al. Arthroscopic evaluation of potential structure modifying activity of hyaluronan (Hyalgan) in osteoarthritis of the knee. Osteoarthritis Cartilage 1997;5:153-60.

26 Corrado EM, Peluso GF, Gigliotti S, De Durante C, Palmieri D, Savoia N, et al. The effects of intra-articular administration of hyaluronic acid on osteoarthritis of the knee: A clinical study with immunological and biochemical evaluations. Eur J Rheumatol Inflamm 1995;15:47-56.

27 Carrabba M, Paresce E, Angelini M, Re KA, Torchiana EEM, Perbellini A. The safety and efficacy of different dose schedules of hyaluronic acid in the treatment of painful osteoarthritis of the knee with joint effusion. Eur J treatment of painful osteoarthritis of
Rheumatol Inflamm 1995;15:25-31.

28 Bucsi L, Poor G. Efficacy and tolerability of oral chondroitin sulphate as a symptomatic slow-acting drug for osteoarthritis (SYSADOA) in the treatment of knee osteoarthritis. Osteoarthritis Cartilage 1998;6(suppl):31-6.
Ubelhart D, Thornar EJM, Delmas PD, Chantraine A, Vignon E. Effects of oral chondroitin sulphate on the progression of knee osteoarthritis: A pilot study. Osteoarthritis Cartilage 1998;6(suppl):39-46. 30 Bourgeois P, Chales G, Dehais J, Delcambre B, Kuntzi JL, Rozenberg S. chondroitin sulphate $3 \times 400 \mathrm{mg} /$ day vs placebo. Osteoarthritis Cartilage 1998;6(suppl):25-30.

31 Morreale P, Manopulo R, Galati M, Boccanera L, Saponati G, Bocchi L. Comparison of the antiinflammatory efficacy of chondroitin sulphate and diclofenac sodium in patients with knee osteoarthritis. J Rheumatol 1996; 23:1385-91.

32 Pujalte JM, Llavore EP, Ylescupidez FR. Double-blind clinical evaluation of oral glucosamine sulphate in the basic treatment of osteoarthrosis. Curr oral glucosamine sulphate in the

33 Noack W, Fischer M, Forster KK, Rovati LC, Setnikar I. Glucosamine sulphate in osteoarthritis of the knee. Osteoarthritis Cartilage 1994;2:51-9.

34 Muller-Fassbender H, Bach GL, Haase W, Rovati LC, Setnikar I. Glucosamine sulphate compared to ibuprofen in osteoarthritis of the knee. Osteoarthritis Cartilage 1994;2:61-9.

35 Gramajo RJ, Cutroneo EJ, Fernandez DE, Gibson JL, Caceres Maldonado JC, Romero FL, et al. A single-blind, placebo-controlled study of glycosaminoglycan-peptide complex ('Rumalon') in patients with osteoarthritis of the hip or knee. Curr Med Res Opin 1989;11:366-73.

36 Pavelka K Jr, Sedlackova M, Gatterova J, Becvar R, Pavelka K Sr. Glycosaminoglycan polysulfuric acid (GAGPS) in osteoarthritis of the knee. Osteoarthritis Cartilage 1995;3:15-23.

37 Superio-Cabuslay E, Ward MM, Lorig KR. Patient education interventions in osteoarthritis and rheumatoid arthritis: a meta-analytic comparison with nonsteroidal antiinflammatory drug treatment. Arthritis Care Research 1996;9:292-301.

38 Keefe FJ, Caldwell DS, Williams DA, Gil KM, Mitchell D, Robertson C, et al. Pain coping skills training in the management of osteoarthritic knee pain: a comparative study. Behavior Therapy 1990;21:49-62.

39 Keefe FJ, Caldwell DS, Williams DA, Gil KM, Mitchell D, Robertson C, et al. Pain coping skills training in the management of osteoarthritic knee al. Pain coping skills training in the management of osteoar
pain-II: follow-up results. Behavior Therapy 1990;21:435-47.

40 Keefe FJ, Caldwell DS, Baucom D, Salley A, Robinson E, Timmons K, et al. Spouse-assisted coping skills training in the management of osteoarthritic knee pain. Arthritis Care Research 1996;9:279-91.

41 Kovar PA, Allegrante JP, MacKenzie CR, Peterson MG, Gutin B, Charlson ME. Supervised fitness walking in patients with osteoarthritis of the knee. A randomized, controlled trial [see comments]. Ann Intern Med 1992;116:529-34.

42 Tohyama H, Yasuda K, Kaneda K. Treatment of osteoarthritis of the knee with heel wedges. Int Orthop 1991;15:31-3.

43 Willims RA, Foulsham BM. Weight reduction in osteoarthritis using phentermine. Practitioner 1981;225:231-2.

44 Felson DT, Zhang Y, Anthony JM, Naimark A, Anderson JJ. Weight loss reduces the risk for symptomatic knee osteoarthritis in women. The Framreduces the risk for symptomatic knee osteoarthritis in women. The
ingham Study [see comments]. Ann Intern Med 1992;116:535-9.

45 Schilke JM, Johnson GO, Housh TJ, O'Dell JR. Effects of muscle-strength training on the functional status of patients with osteoarthritis of the knee joint. Nurs Res 1996;45:68-72.

46 Rogind H, Bibow-Nielsen B, Jensen B, Moller HC, Frimodt-Moller H, Bliddal $\mathrm{H}$. The effects of a physical training program on patients with osteoarthritis of the knees. Arch Phys Med Rehabil 1998;79:1421-7.

47 Knutson K, Lewold S, Lidgren L, Robertson O. The Swedish knee arthroplasty register. A nationwide study of 30,003 knees 1976-1992. Acta Orthop Scand 1994;65:375-86.

48 Field MJ, Lohr KN. Clinical practice guidelines: directions for a new program. Washington DC: National Academy Press, 1990.

49 Woolf SH, Grol R, Hutchinson A, Eccles M, Grimshaw J. Clinical guidelines: potential benefits, limitations, and harms of clinical guidelines. BMJ 1999;318:527-30.

50 Juni P, Witschi A, Bloch R, Egger M. The hazards of scoring the quality of clinical trials for meta-analysis [see comments]. JAMA 1999;282:1054-60.

51 Doherty M, Jones A. Design of clinical trials in knee osteoarthritis: practical issues for debate. Osteoarthritis Cart 1998;6:371-3. 